\title{
Candidemia in Internal Medicine Wards: a real problem or just
} an epiphenomenon?

\author{
Matteo Bassetti, ${ }^{1}$ Alessia Cargnelutti, ${ }^{1}$ Federico Silvestri $^{2}$ \\ ${ }^{1}$ Infectious Diseases Division, Santa Maria Misericordia University Hospital, Udine; ${ }^{2}$ Division of Internal Medicine, General \\ Hospital, Latisana (UD), Italy
}

The global incidence of candidemia raised fivefold in the past 10 years and Candida spp. is currently between the fourth and the sixth most common nosocomial bloodstream isolate in American and European studies. ${ }^{1,2}$ Candida is an emerging cause of bloodstream infections in Internal Medicine Wards (IMWs) and represents an important cause of mortality and morbidity in this setting. Patients with candidemia in IMWs show a particularly high crude mortality rate (ranging between $42 \%$ and $50 \%$ ), even superior than in Intensive Care Units (ICU) and hemato-oncology wards. ${ }^{3}$ Historically, the surveillance studies have demonstrated that the majority of cases of candidemia occurred among those patients admitted to ICU and surgical departments. ${ }^{2,4}$ More recently, studies from different countries including Italy, have shown that the great majority of nosocomial cases occur among patients in IMWs in tertiary care hospitals. ${ }^{5-7} \mathrm{~A}$ recent Italian report by Silvestri et al. (in the current issue of

Correspondence: Matteo Bassetti, Divisione Malattie Infettive, AOU Santa Maria della Misericordia, p.le Santa Maria della Misericordia 15, 33100 Udine, Italy.

Tel.: +39.0432.559355 - Fax: +39.0432.559360.

E-mail:mattba@tin.it

Key words: candidemia, internal medicine wards, de-escalation therapy.

Conflict of interests: MB serves on scientific advisory boards for Pfizer Inc., MSD, Gilead and Astellas Pharma Inc.; he received funding for travel or speaker honoraria from the same companies (Pfizer Inc., MSD, Gilead Sciences and Astellas Pharma Inc.). Other authors declare no potential conflict of interests.

Received for publication: 24 April 2014.

Accepted for publication: 22 May 2014.

This work is licensed under a Creative Commons Attribution NonCommercial 3.0 License (CC BY-NC 3.0).

CCopyright M. Bassetti et al., 2014

Licensee PAGEPress, Italy

Italian Journal of Medicine 2014; 8:205-207

doi:10.4081/itjm.2014.510 this journal) has underlined that many candidemia episodes occur also in district hospitals. ${ }^{8}$ Nevertheless, only few data are available on the epidemiology and the clinical aspects of candidemia in IMWs. In this setting, the reasons for a high incidence of candidemia can be multiple. One hypothesis is that the risk factors associated with the development of candidemia are very common in IMWs. Furthermore, an increase in the elderly population has been registered in IMWs. ${ }^{3}$

Based on the limited number of available studies, patients admitted to IMWs show peculiar demographic characteristics and comorbidities compared to other wards. In particular, mean age is higher than in other settings, with the majority of patients older than 75 years; they display higher APACHE II scores, more solid tumors, higher frequencies of antibiotic therapy prior to candidemia, and a higher number of urinary or central venous catheters and parenteral nutrition. ${ }^{3,8}$

As far as diagnosis is concerned, blood culture represents the gold standard and therefore should be performed every time candidemia is suspected. However, the sensitivity of blood cultures ranges between $50 \%$ and $75 \%$, and the identification time of Candida species is often prolonged. ${ }^{9}$ For these reasons, blood cultures cannot always be considered as a reliable and rapid diagnostic test. The (1,3)- $\beta$-D-glucan (BG), a fungal biomarker, could be useful for the early diagnosis of candidemia in patients at high risk. Several studies reported that among critically ill patients with proven or probable fungal infection, BG became detectable prior to the onset of clinical symptoms and blood cultures positivity. ${ }^{10} \mathrm{BG}$ is recommended in several guidelines for early diagnosis of invasive candidiasis. ${ }^{11,12}$ Nevertheless, there is no evidence that BG can be used to achieve the diagnosis of candidemia in IMWs yet, and further studies are required. According to the last recommendations, it is reasonable to use $\mathrm{BG}$ as an adjunctive marker in order to obtain a rapid diagnosis of candidemia, taking also in mind that the BG sensitivity is very high in ruling out fungal infection if the test results negative. ${ }^{13}$

Regarding the antifungal treatment, several studies have demonstrated that the time from the first positive blood culture drawn to the initiation of therapy correlates with an increase in mortality. ${ }^{14,15}$ Recent data con- 
firm that the timing of the start of antifungal therapy may be a key factor to impact mortality among patients with candidemia. In a recent retrospective study only $7.3 \%$ of patients treated in IMWs received antifungal treatment within $48 \mathrm{~h}$, while the rate of patients treated within $48 \mathrm{~h}$ was $25.5 \%$ in other wards $(\mathrm{P}<0.001)$. Since the mortality rate was lower among patients receiving an adequate antifungal therapy within $48 \mathrm{~h}$, it is reasonable to assume that a delay in starting antifungal therapy is an important factor involved in poor outcomes. ${ }^{3}$

One of the reasons why a delay is often seen in the administration of antifungal treatment can be related to the lack of awareness of candidemia in the daily clinical practice in IMWs. Another reason could be that, in some cases, candidemia represents one of the several complications occurring during hospitalization rather than the main cause of mortality. Thus, in certain instances it is difficult to state if the patient died with candidemia or due to candidemia.

Recent guidelines no longer consider fluconazole as the drug of choice for invasive candidiasis, especially in moderate to severely ill patients. ${ }^{11}$ The rationale is based on the increasing prevalence of Candida species with decreased susceptibility to fluconazole and the lower clinical efficacy of fluconazole compared to anidulafungin in patients with candidemia and invasive candidiasis. ${ }^{16}$ In regard to Candida infections, all three echinocandins (caspofungin, micafungin and anidulafungin) are fungicidal, exhibit a broad spectrum of activity, are active against biofilm and acquire resistance is rare. At present, all echinocandins are considered drugs of choice for candidemia. Unfortunately, due to their high costs, all suspected cases of candidemia occurring in the internal medicine setting could not be treated with this class of antifungals.

One potential solution would be the de-escalation approach, based on the use of an echinocandin as initial therapy, when the patient is clinically unstable and the diagnosis of candidemia is uncertain, followed by a shift to fluconazole within few days if the patient is clinically stable and the microbiology tests demonstrate the susceptibility to this drug or, another solution would be to stop the antifungal treatment if the BG values are not significant.

This approach requires a close collaboration with the laboratory and the availability of susceptibility testing that may not always be available. Not to say that currently BG testing is still not very widespread.

Fluconazole is a well-tolerated drug without significant toxicity, but exhibits an important pharmacokinetic variability depending on the patient's characteristics and comorbidities. Moreover, azoles are substrates for CYP450 enzymes and thus may show relevant drug-drug interactions. Patients admitted to IMWs are typically characterized by multiple organ dysfunctions and concomitant drugs; for this reason therapeutic drug monitoring might be considered to optimize therapy and avoid potential toxicity.

In conclusion the increased prevalence of candidemia does not seem just an epiphenomenon, but a real problem that affects morbidity, mortality and hospital costs.

\section{References}

1. Wisplinghoff H, Bischoff T, Tallent SM, et al. Nosocomial bloodstream infections in US hospitals: analysis of 24,179 cases from a prospective nationwide surveillance study. Clin Infect Dis 2004;39:309-17.

2. Marchetti O, Bille J, Fluckiger U, et al. Epidemiology of candidaemia in Swiss tertiary care hospitals: secular trends, 1991-2000. Clin Infect Dis 2004;38:311-20.

3. Bassetti M, Molinari MP, Mussap M, et al. Candidaemia in internal medicine departments: the burden of a rising problem. Clin Microbiol Infect 2013;19:281-4.

4. Pfaller MA, Jones RN, Doern GV, et al. International surveillance of blood stream infections due to Candida species in the European SENTRY Program: species distribution and antifungal susceptibility including the investigational triazole and echinocandin agents. SENTRY Participant Group (Europe). Diagn Microbiol Infect Dis 1999;35:19-25.

5. Bassetti M, Merelli M, Righi E, et al. Epidemiology, species distribution, antifungal susceptibility, and outcome of candidemia across five sites in Italy and Spain. J Clin Microbiol 2013;51:4167-72.

6. Motta AL, Almeida GM, Almeida Júnior JN, et al. Candidemia epidemiology and susceptibility profile in the largest Brazilian teaching hospital complex. Bras J Infect Dis 2010;14:441-82.

7. Spiliopoulou A, Vamvakopoulou S, Bartzavali C, et al. Eleven-year retrospective survey of candidaemia in a university hospital in southwestern Greece. Clin Microbiol Infect 2010;16:1378-81.

8. Silvestri F, Bassetti M, Zaramella M, et al. Epidemiology, species distribution, antifungal susceptibility and outcome of candidemia among Internal Medicine Wards of community hospitals of Udine province, Italy. Ital J Med 2014; 8:253-8.

9. Meyer MH, Letscher-Bru V, Jaulhac B, et al. Comparison of mycosis IC/F and plus aerobic/F media for diagnosis of fungemia by the bactec 9240 system. J Clin Microbiol 2004;42:773-7.

10. Tissot F, Lamoth F, Hauser PM, et al. $\beta$-glucan antigenemia anticipates diagnosis of blood culture-negative intraabdominal candidiasis. Am J Respir Crit Care Med 2013;188:1100-9.

11. Cornely OA, Bassetti M, Calandra T, et al. ESCMID* guideline for the diagnosis and management of Candida diseases 2012: non-neutropenic adult patients. Clin Microbiol Infect 2012;18:19-37.

12. Bassetti M, Marchetti M, Chakrabarti A, et al. A research agenda on the management of intra-abdominal candidiasis: results from a consensus of multinational experts. Intensive Care Med 2013;39:2092-106.

13. Eggimann P, Marchetti O. Is $(1 \rightarrow 3)-\beta$-D-glucan the miss- 
ing link from bedside assessment to pre-emptive therapy of invasive candidiasis? Crit Care 2011;15:1017.

14. Morrell M, Fraser VJ, Kollef MH. Delaying the empiric treatment of candida bloodstream infection until positive blood culture results are obtained: a potential risk factor for hospital mortality. Antimicrob Agents Chemother 2005;49:3640-5.

15. Garey KW, Rege M, Pai MP, et al. Time to initiation of fluconazole therapy impacts mortality in patients with candidemia: a multi-institutional study. Clin Infect Dis 2006;43:25-3.

16. Kett DH, Shorr AF, Reboli AC, et al. Anidulafungin compared with fluconazole in severely ill patients with candidemia and other forms of invasive candidiasis: support for the 2009 IDSA treatment guidelines for candidiasis. Crit Care 2011;15:R253. 